\title{
World Health Organization treatment guidelines for drug-resistant tuberculosis, 2016 update
}

\author{
Dennis Falzon ${ }^{1}$, Holger J. Schünemann², Elizabeth Harausz ${ }^{3}$, \\ Licé González-Angulo ${ }^{1}$, Christian Lienhardt ${ }^{1}$, Ernesto Jaramillo ${ }^{1}$ and \\ Karin Weyer ${ }^{1}$
}

Affiliations: ${ }^{1}$ Global TB Programme, World Health Organization, Geneva, Switzerland. ${ }^{2}$ McMaster University Health Sciences Centre, Hamilton, ON, Canada. ${ }^{3}$ US Military HIV Research Program, Walter Reed Army Institute of Research, Silver Spring, MD, USA.

Correspondence: D. Falzon, Global TB Programme, World Health Organization, 20 Avenue Appia, 1211 Geneva 27, Switzerland. E-mail: falzondawho.int

@ERSpublications

The latest WHO recommendations for the treatment of multidrug- and rifampicin-resistant tuberculosis (MDR/RR-TB) http://ow.ly/Lj5K307XZ7h

Cite this article as: Falzon D, Schünemann HJ, Harausz E, et al. World Health Organization treatment guidelines for drug-resistant tuberculosis, 2016 update. Eur Respir J 2017; 49: 1602308 [https://doi.org/ 10.1183/13993003.02308-2016].

ABSTRACT Antimicrobial resistance is a major global concern. Tuberculosis (TB) strains resistant to rifampicin and other TB medicines challenge patient survival and public health. The World Health Organization (WHO) has published treatment guidelines for drug-resistant TB since 1997 and last updated them in 2016 based on reviews of aggregated and individual patient data from published and unpublished studies. An international expert panel formulated recommendations following the GRADE approach. The new WHO guidelines recommend a standardised 9-12 months shorter treatment regimen as first choice in patients with multidrug- or rifampicin-resistant TB (MDR/RR-TB) strains not resistant to fluoroquinolones or second-line injectable agents; resistance to these two classes of core second-line medicines is rapidly detectable with molecular diagnostics also approved by WHO in 2016. The composition of longer regimens for patients ineligible for the shorter regimen was modified. A first-ever meta-analysis of individual paediatric patient data allowed treatment recommendations for childhood MDR/RR-TB to be made. Delamanid is now also recommended in patients aged 6-17 years. Partial lung resection is a recommended option in MDR/RR-TB care. The 2016 revision highlighted the continued shortage of high-quality evidence and implementation research, and reiterated the need for clinical trials and best-practice studies to improve MDR/RR-TB patient treatment outcomes and strengthen policy.

This article has supplementary material available from erj.ersjournals.com

Earn CME accreditation by answering questions about this article. You will find these at erj.ersjournals.com/journal/cme Received: Nov 242016 | Accepted after revision: Jan 032017

Support statement: This study was supported by the US Agency for International Development USAID-WHO Consolidated Grant No. GHA-G-00-09-0. Funding information for this article has been deposited with the Open Funder Registry.

Conflict of interest: Disclosures can be found alongside this article at erj.ersjournals.com

This is one of a selection of articles published as ERJ Open papers, as part of an initiative agreed between the European Respiratory Society and the World Health Organization.

The content of this work is copyright of the authors or their employers. Design and branding are copyright @ERS 2017. This version is distributed under the terms of the Creative Commons Attribution Non-Commercial Licence 4.0. 


\section{Introduction}

Antimicrobial resistance has become a topical health and security concern for countries worldwide [1]. In the course of the last 20 years it has become increasingly clear that global efforts to end tuberculosis (TB) will continue to face a major challenge with the widespread dissemination of $\mathrm{TB}$ strains that are resistant to medicines used in its treatment $[2,3]$. Unlike the large majority of TB patients worldwide who can expect a relapse-free cure with a 6-month course of first-line medication [4], patients with rifampicin-resistant $\mathrm{TB}$ (RR-TB), commonly combined with isoniazid resistance (multidrug-resistant TB (MDR-TB)), require treatment regimens which are longer, less effective and less accessible than first-line regimens, but more costly, toxic and complicated to deliver. Most second-line MDR-TB regimens in recent decades were designed to last $\geqslant 20$ months [5], presenting a formidable challenge to health service providers to ensure patient adherence and cure rates.

The World Health Organization (WHO) estimates that 3.9\% (95\% CI 2.7-5.1\%) of previously untreated and $21 \%$ (95\% CI 15-28\%) of previously treated TB cases occurring worldwide in 2015 had MDR/RR-TB [6]. Each year about 580000 (range 520000-640000) new MDR/RR-TB cases emerge among new and retreated TB patients, and cause about 250000 (range 160000-340000) deaths. Globally, 51\% (95\% CI $30-70 \%)$ of MDR-TB patients have strains with additional resistance to fluoroquinolones or second-line injectable agents, critical agents of any second-line MDR-TB treatment regimen, while 9.5\% (95\% CI 7.0-12\%) are resistant to both of these classes of medicines (extensively drug-resistant TB (XDR-TB)) [6].

Although countries have been placing more patients on MDR/RR-TB treatment over the last years, only about 125000 patients of the approximately 580000 new cases were reported to have been started on second-line TB treatment regimens globally in 2015 [6]. Little is known about the quality of care that these patients, as well as many others who are not reported, received. However, in recent years, national TB programmes reported that only about a half of patients with MDR/RR-TB and a fourth of those with XDR-TB had a successful treatment outcome [6].

A number of hurdles stand in the way of mounting an effective global response to drug-resistant TB [7]. A recent survey of TB diagnostic and treatment practices in 24 countries identified suboptimal implementation of policies which have an important bearing on drug-resistant TB care and prevention, including the use of rapid diagnostics, the roll-out of effective treatment regimens for both drug-susceptible and MDR-TB, and regulatory frameworks (e.g. updating essential medicines lists) [8]. Insufficient political will to fund health systems adequately and the shortage of skilled health workers to diagnose and manage drug-resistant TB are likely to contribute to these bottlenecks [9]. The adoption of effective second-line TB treatment regimens must include appropriate patient support as part of a comprehensive long-term solution [10].

Guidance on TB treatment forms part of WHO's core mandate to support national TB programmes in broadening access to care, within the larger set of objectives established by the End TB Strategy for the post-2015 setting $[11,12]$. Since 1997, the Global TB Programme of WHO (WHO/GTB) has regularly issued guidelines and implementation manuals for the diagnosis and treatment of drug-resistant TB [5, 13-28]. Here, we describe the latest update in WHO policies on the treatment of drug-resistant TB and discuss the implications of the changes for implementers in the coming years.

\section{Material and methods}

Preparation for the guidelines revision

Since 2011, WHO guidelines for the treatment of drug-resistant TB have been developed following the GRADE approach (Grading of Recommendations Assessment, Development and Evaluation; www. gradeworkinggroup.org), in line with the WHO's requirements for evidence review and policy development $[29,30]$. GRADE defines a logical sequence of steps to develop evidence-based clinical and policy guidance. The process starts with a scoping of priority policy issues, the translation of these priorities into structured questions, and a systematic search, review and quality assessment of the evidence for the questions (table 1). Independent experts formulate the guideline recommendations informed by evidence summaries and considerations such as feasibility, values and preference and equity [31, 32].

The update of the 2016 guidelines started in late 2014 with a prioritisation of the policy and practice areas for update. WHO invited an international team of experts with a broad technical knowledge base to form part of a Guideline Development Group (GDG). The GDG membership represented an extensive cross-section of geographic settings and health systems, future users of the guidelines as well as affected persons (including a patient). A second group composed of national TB programme staff, clinicians and public health experts was appointed to serve in a peer-review capacity (External Review Group (ERG)). Ahead of their appointments to serve in these groups, the experts completed a declaration of interest, which guided their inclusion in the GDG and ERG and/or their participation in specific topics during the guideline development [25]. A number of online webinars were held with both the GDG and the ERG before the physical guidelines meeting held in Geneva, Switzerland on November 9-11, 2015. 
TABLE 1 Certainty of the evidence and definitions

\begin{tabular}{|c|c|}
\hline $\begin{array}{l}\text { Certainty of the } \\
\text { evidence }\end{array}$ & Definition \\
\hline High $(\oplus \oplus \oplus \oplus)$ & Further research is very unlikely to change our confidence in the estimate of effect \\
\hline Moderate $(\oplus \oplus \oplus \circ)$ & $\begin{array}{l}\text { Further research is likely to have an important impact on our confidence in the } \\
\text { effect and may change the estimate }\end{array}$ \\
\hline Low $(\oplus \oplus \circ))$ & $\begin{array}{l}\text { Further research is very likely to have an important impact on our confidence in the } \\
\text { estimate of effect and is likely to change the estimate }\end{array}$ \\
\hline Very low $(\oplus \circ \bigcirc)$ ) & Any estimate of effect is very uncertain \\
\hline
\end{tabular}

\section{Setting the scope of the updated guidelines}

The scope of the 2016 guidelines update covered the following areas: 1) the optimal combination of medicines and approach towards regimen design for TB patients (both adults and children) with RR-TB, MDR-TB, XDR-TB and isoniazid-resistant TB, as well as for patients with Mycobacterium bovis disease; 2) the effectiveness and safety of standardised regimens lasting up to 12 months for the treatment of patients with MDR-TB ("shorter regimens") when compared with longer conventional treatment; 3 ) the effect of time to start of treatment on drug-resistant TB patient outcomes; and 4) the effect of surgical interventions on treatment outcomes for patients with drug-resistant TB.

The scope of the May 2016 update of the guidelines excluded aspects of the programmatic management of drug-resistant TB for which no substantive new evidence had emerged since the 2011 revision of WHO policy guidance on drug-resistant $\mathrm{TB}$, i.e. the testing for rifampicin resistance, the monitoring of treatment response, the duration of conventional regimens, the start of antiretroviral therapy in MDR-TB patients with HIV infection and models of care. New data on bedaquiline and delamanid use within longer regimens were reviewed by a separate GDG process from June 2016, subsequent to the release of the treatment guidelines in May 2016 [27, 33].

The guideline scope was expressed in four structured questions using the GRADE-recommended PICO format (Population, Intervention, Comparator to the intervention and Outcomes) (supplementary table S1) [30]. All relevant outcomes for these questions were scored in the critical range by the GDG members (table 2). For the purposes of the review, a serious adverse event was classified as either Grade 3 (severe) or Grade 4 (life-threatening or disabling), or which led to the medicine being stopped permanently.

\section{Review of evidence}

Expert reviewers were commissioned by WHO from academic centres to assess the evidence for the PICO questions through systematic literature reviews following a standard methodology [34] (see Acknowledgements). Titles, abstracts and the full text of potentially relevant literature were screened using key subject words and text words. Authors in the field and members of the GDG were contacted to identify missing studies or studies in progress. In addition to aggregated data, individual patient-level data (IPD) were collected and analysed to inform the recommendations on the shorter MDR-TB regimen, longer regimens in children and adults [16],

\section{TABLE 2 What are the most important outcomes to consider when making decisions on the} treatment of drug-resistant tuberculosis (TB)?

\begin{tabular}{lr} 
Outcomes & Mean scor \\
\hline Adherence to TB treatment (treatment interruption due to nonadherence) & 6.8 \\
Avoiding adverse reactions from TB drugs & 7.0 \\
Avoiding the acquisition or amplification of drug resistance & 7.9 \\
Cure or successful completion by end of treatment & 9.0 \\
Culture conversion by month 6 & 7.4 \\
Death (survival) by the end of projected treatment & 8.1 \\
Treatment failure & 8.7 \\
Relapse & 7.7
\end{tabular}

Members of the Guideline Development Group scored TB outcomes according to their relative priority when making decisions on drug-resistant TB treatment. They were asked to take a societal perspective in rating the outcomes. 1-3 points: not important for making recommendations on drug-resistant TB treatment; 4-6 points: important but not critical for making recommendations on drug-resistant TB treatment; 7-9 points: critical for making recommendations on drug-resistant TB treatment. 
and the use of surgery [17]. Supplementary table S2 summarises the evidence reviews done for each PICO question.

Estimates of effect were calculated from the pooled data of studies included. Where possible, adjustments were made to reduce confounding and other bias. GRADE evidence profiles, based on the results of systematic reviews, were prepared for each question using the GRADEpro online application (www.gradepro.org). The quality of evidence was assessed using the following criteria: study design, limitations in the studies (risk of bias), imprecision, inconsistency, indirectness, publication bias, magnitude of effect, dose-effect relations and residual confounding. GRADE evidence profiles were discussed by the GDG before formulating the recommendations. The group used the "Evidence to Decision" tables via the GRADEpro interface to capture the content of the discussions, annotate the different considerations, develop the wording and strength of the recommendations, and add the remarks that accompany each recommendation [25]. Apart from the quality of evidence, the strength of a recommendation was also determined by assessing the balance between desirable and undesirable effects, values and preferences, considerations on equity, resource use and feasibility. The GDG gave particular importance to the following guiding principles: 1) the promotion of universal access to MDR-TB care and equity in low-resource settings, 2) prevention of death and transmission of drug-resistant TB through early diagnosis, 3) avoidance of harm by ascertaining resistance patterns before starting treatment and active monitoring for and management of drug-related toxicity during treatment [35], and 4) provision of care in a setting acceptable to the patient.

\section{Results}

\section{Evidence reviews}

Shorter MDR-TB regimen

Evidence used to assess shorter MDR-TB regimens was derived from observational studies in 10 countries [36]. The analysis included IPD from Bangladesh $(n=493)$ [37], Swaziland $(n=24)$ and Uzbekistan $(n=65)$, as well as aggregated from seven sub-Saharan African countries $(n=408$; Benin, Burkina Faso, Burundi, Cameroon, Central African Republic, Democratic Republic of the Congo and Niger) [38]. Additional aggregated data from Cameroon $(n=150)$ [39] and Niger $(n=65)$ [40] were also used. The outcomes of patients on shorter regimens were compared with those of 7665 MDR-TB patients treated with "conventional" longer regimens who had not been previously exposed to second-line TB medicines (IPD were available for these patients) [41]. In these analyses, previously untreated patients receiving the shorter MDR-TB treatment regimens had a higher likelihood of treatment success than those who received longer regimens: $84 \%$ (95\% CI 79-87\%) versus 62\% (95\% CI 53-70\%) when compared with treatment failure/ relapse/death/loss to follow-up. Relapses following the shorter regimens were rare, although this may be due to the relatively small number of patients and the limited duration of follow-up after completing treatment. Interim findings from the multicentre study in Francophone African countries used in this analysis did not detect any relapse among 39\% of patients followed up 12-18 months after treatment termination, most of whom were also confirmed to have a negative culture. Treatment success was lower among the small number of patients with additional resistance to pyrazinamide and/or fluoroquinolones on shorter MDR-TB regimens, even if in general it remained higher than in patients on longer regimens. Adverse event reporting (classification, completeness) differed between the cohorts. Hyperglycaemia was reported from two out of three sites which used high-dose gatifloxacin $(<10 \%$ of patients exposed [ 40 , 42]). Hearing loss was the most common, potentially serious adverse event and was detected in $43 \%$ of patients assessed in Cameroon, half of whom experienced a substantial impairment [39].

\section{Longer MDR-TB regimen (adults)}

With the exception of three randomised controlled trials (RCTs) for clofazimine and linezolid [43-45], the evidence used to assess the effects of individual medicines on the outcomes of adult patients on longer MDR-TB regimens originated from observational studies. Meta-analysis were conducted on two datasets: 1) an IPD dataset compiled from studies of 9153 patients treated between the early 1980s and 2009 [41], and 2) a study-level aggregated dataset for over 17000 MDR-TB and XDR-TB patients in 74 studies published between January 2009 and August 2015 (BASTOS M, LAN Z, MENZIES R, personal communication). Subjects included in the latter analysis had similar treatment success rates to those in the IPD (60\% in MDR-TB and $26 \%$ in XDR-TB) and the frequency of serious adverse events ranged from $0.5 \%$ to $12.2 \%$. For serious adverse events, reference was also made to another recent review on the safety of certain second-line TB medicines [46]. There was important variation across studies in the way adverse events were elicited (passive versus active), the completeness of their reporting, and the criteria and methods used to assign severity, seriousness and organ class coding. In the case of high-dose isoniazid, the effects from the paediatric IPD meta-analysis were applied to adults. 
Longer MDR-TB regimen (children)

The evidence used to assess longer MDR-TB regimens in children was derived from published and unpublished data of observational studies collected until September 30, 2014. 27 eligible studies provided IPD, allowing the pooling of records from 974 cases ( 36 children with XDR-TB were excluded from further analysis). The overall treatment success was $80 \%$, with $9 \%$ deaths and $10 \%$ lost to follow-up. The analyses for the determinants of treatment success were undertaken separately for children who had bacteriologically confirmed MDR-TB ( $\mathrm{n}=701 ; 9 \%$ with strains resistant to a fluoroquinolone or a second-line injectable agent) and those who were only clinically diagnosed with MDR-TB ( $n=237)$. Children without malnutrition, not showing advanced disease on chest radiography, without severe forms of extrapulmonary disease or HIV-negative were considered to have milder forms of disease. Severe forms of disease were associated with bacteriologically confirmed MDR-TB. When data on the use of older MDR-TB medicines in children were inconclusive or unavailable, evidence from adults was extrapolated to children.

Surgery

Evidence for the effectiveness of different forms of elective pulmonary surgery in MDR-TB patients was derived from an IPD meta-analysis of 26 cohorts, as well as a systematic review and study-level meta-analysis $[47,48]$. Two sets of analyses were undertaken: for partial pulmonary resection and for radical pneumonectomy. The study-level meta-analysis that examined all forms of surgery together reported that patients who received surgery were more likely to complete their treatment successfully. However, the IPD meta-analysis showed that patients who underwent partial lung resection had a higher likelihood of treatment success than those who did not undergo surgery (odds ratio (OR) 3.5, 95\% CI 1.5-8.1), which was not the case in patients who had a radical pneumonectomy. The risk of death in the IPD did not differ between patients who underwent partial lung resection and those who received medical treatment only (OR 0.6, 95\% CI 0.2-2.2). However, these outcomes could be biased because the risk of death could have been much higher among patients in whom surgery was prescribed had they not been operated upon. There were not enough data on adverse events, surgical complications or long-term sequelae to allow a meaningful analysis. Treatment success in XDR-TB patients was lower when patients underwent surgery compared with patients who did not (OR $0.4,95 \%$ CI $0.2-0.9$ ), an effect that is likely to be biased by accompanying factors which predisposed to poor outcomes in patients who underwent surgery and which could not be adjusted for.

Other

The two reviews on the treatment of isoniazid-resistant TB and on the time to start of MDR-TB treatment could not trace evidence to address the guideline questions [49]. There were very few published studies on the treatment of Mycobacterium bovis and the regimens used differed substantially, precluding any attempt at formulating recommendations for clinical use. A separate review of newly available data on the use of bedaquiline was undertaken following the release of the updated drug-resistant TB treatment guidelines in May 2016 [33]. Similarly, a review of data on the use of delamanid in the treatment of MDR-TB in children aged 6-17 years informed a new recommendation issued in October 2016 (see also remarks underneath Recommendation 4 below) [27].

\section{Regrouping, recommendations and remarks}

The grouping of medicines used for the treatment of MDR/RR-TB and XDR-TB was revised on the basis of the GDG discussions of the updated evidence reviews for effectiveness and safety (table 3). The medicines making up the "core" second-line components of a longer MDR-TB regimen are now classified in four groups (A, B, C and D). Group D is divided into three subgroups, i.e. first-line anti-TB medicines (D1), bedaquiline and delamanid (D2), and other agents of uncertain role in MDR-TB treatment (D3). WHO considers that currently only the medicines in these four groups are relevant to MDR-TB treatment regimens under programmatic conditions.

The certainty in the evidence reviewed to address the PICO questions was rated as very low and all five recommendations proposed by the GDG were graded as conditional.

Eligibility for MDR-TB regimen

1) Any patient (child or adult) with RR-TB in whom isoniazid resistance is absent or unknown may be treated with a recommended MDR-TB regimen, either a shorter MDR-TB regimen, or if this cannot be used, a longer MDR-TB regimen to which isoniazid is added.

Remarks: This applies to patients with rifampicin monoresistance or non-MDR-TB polyresistance. It implies that TB patients with strains found to be rifampicin resistant when tested with Xpert MTB/RIF, line probe assay (LPA) or conventional diagnostics need to start a recommended MDR-TB treatment regimen irrespective of whether isoniazid resistance is undetermined or confirmed. This recommendation 
TABLE 3 General steps in designing the composition of a longer multidrug-resistant tuberculosis (MDR-TB) regimen ${ }^{\#}$

\begin{tabular}{|c|c|c|}
\hline \multirow[t]{2}{*}{ Step } & \multicolumn{2}{|r|}{ Medicines } \\
\hline & Grouping & Options \\
\hline \multirow{3}{*}{ 1. Add one later-generation fluoroquinolone } & $A^{\pi}$ & Levofloxacin (Lfx) \\
\hline & & Moxifloxacin (Mfx) \\
\hline & & Gatifloxacin (Gfx) \\
\hline \multirow[t]{4}{*}{ 2. Add one second-line injectable agent } & $\mathrm{B}$ & Amikacin (Am) \\
\hline & & Capreomycin (Cm) \\
\hline & & Kanamycin (Km) \\
\hline & & (Streptomycin) (S) ${ }^{+}$ \\
\hline \multirow[t]{4}{*}{ 3. Add two or more second-line agents } & $C^{\pi}$ & Ethionamide/prothionamide (Eto/Pto) \\
\hline & & Cycloserine/terizidone (Cs/Trd) \\
\hline & & Linezolid (Lzd) \\
\hline & & Clofazimine (Cfz) \\
\hline \multirow{3}{*}{$\begin{array}{l}\text { 4. Add pyrazinamide and any other first-line agent } \\
\text { (if they can help strengthen the regimen) }\end{array}$} & D1 & Pyrazinamide (Z) \\
\hline & & Ethambutol (E) \\
\hline & & High-dose isoniazid $\left(\mathrm{H}^{\mathrm{h}}\right)$ \\
\hline \multirow[t]{2}{*}{ 5. Add bedaquiline or delamanid } & $\mathrm{D} 2$ & Bedaquiline $(B d q)^{\S}$ \\
\hline & & Delamanid (DIm) $)^{\S}$ \\
\hline \multirow{4}{*}{$\begin{array}{l}\text { 6. Add any of these agents (if the regimen cannot } \\
\text { be composed otherwise) }\end{array}$} & D3 & p-aminosalicylic acid (PAS) \\
\hline & & Imipenem-cilastatin (Ipm-Cln) ${ }^{f}$ \\
\hline & & Meropenem $(\mathrm{Mpm})^{f}$ \\
\hline & & $\begin{array}{l}\text { Amoxicillin-clavulanate }(\mathrm{Amx}-\mathrm{Clv})^{f} \\
\text { (Thioacetazone) }(\mathrm{T})^{\# \#}\end{array}$ \\
\hline
\end{tabular}

\#: this stepwise approach guides the design of longer individualised regimens for patients who are not eligible for the World Health Organization-recommended shorter regimen (the composition of the shorter MDR-TB regimen is standardised) (see main text). The aim is to combine at least five effective agents in the intensive phase; more medicines may be included if they can safely increase the chances of cure. The choice of a medicine is based on the likelihood of its effectiveness, on reliable information on drug resistance and on the balance of expected benefits to risk. For instance, in case of nephrotoxicity or hearing loss, an injectable agent may be omitted and additional agents from Group C or D2 included. ๆ: medicines in Groups $\mathrm{A}$ and $\mathrm{C}$ are shown in decreasing order of preference for use (subject to other considerations). ${ }^{+}$: streptomycin may substitute other injectable agents when the other three cannot be used; resistance to streptomycin alone does not qualify for the definition of extensively drug-resistant TB [50]. §: bedaquiline or delamanid may be added to the longer regimen to replace another second-line agent or to strengthen it in accordance with the interim policies [20, 21, 27]. $f$ : carbapenems and clavulanate are used together; clavulanate is only available in formulations combined with amoxicillin. \#\# : HIV status must be tested and confirmed to be negative before thioacetazone is started.

thus supersedes previous advice on the treatment of such cases, such as the replacement of rifampicin with a fluoroquinolone in a first-line TB regimen.

\section{Shorter MDR-TB regimen}

2) In patients with MDR/RR-TB who were not previously treated with second-line drugs and in whom resistance to fluoroquinolones and second-line injectable agents was excluded or is considered highly unlikely, a shorter MDR-TB regimen of 9-12 months may be used instead of the longer regimens.

Remarks: The shorter MDR-TB regimen is the treatment option of first choice for MDR/RR-TB patients, subject to eligibility. Figure 1 summarises the main factors to consider when deciding. The regimen may be used in children and in patients on antiretroviral agents, but it is not indicated if strains have (or are highly likely to have) resistance to medicines in the regimen (except isoniazid). All efforts should be made to exclude resistance to fluoroquinolones and to injectable agents in the regimen before starting treatment, preferably by using in vitro testing with molecular or phenotypic methods. Implementing this recommendation is facilitated by WHO's concurrent endorsement in May 2016 of LPA tests to detect resistance to fluoroquinolones and second-line injectable agents [28]. Experience with the use of the shorter MDR-TB regimen remains limited. It is not indicated in pregnancy (the aminoglycosides and thiamides may be replaced with other medicines in a longer regimen) nor in patients with extrapulmonary forms of disease. The composition and duration of the regimen are fairly standardised and changes are only permissible within the limits of the modifications made in the studies [51]. Programmes are expected to implement a schedule for regular patient monitoring for the whole duration of treatment and also to 


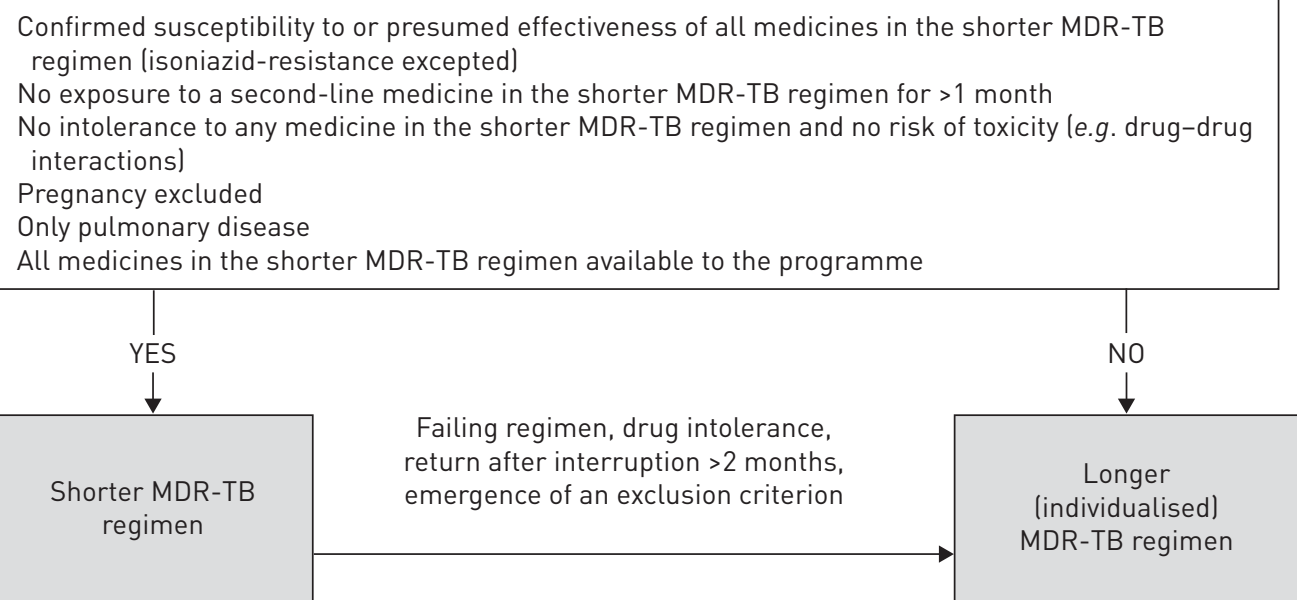

FIGURE 1 Choosing the treatment regimen in patients with confirmed multidrug- and rifampicin-resistant TB (MDR/RR-TB).

control for relapse following the end of treatment (e.g. [52]). There are no data on the use of bedaquiline or delamanid with a shorter MDR-TB regimen and therefore no recommendation can be made in this respect.

\section{Longer MDR-TB regimens}

3) In patients with MDR/RR-TB, a regimen with at least five effective TB medicines during the intensive phase may be used, including pyrazinamide and four second-line TB medicines: one chosen from Group A, one from Group B and at least two from Group C (see table 3 for the composition of these medicine groups). If the minimum number of effective TB medicines cannot be composed as given above, an agent from Group D2 and other agents from Group D3 may be added.

4) In patients with MDR/RR-TB the regimen may be further strengthened with high-dose isoniazid and/or ethambutol.

Remarks: In patients who are not eligible for a shorter MDR-TB regimen, a longer MDR-TB regimen is prescribed. The principles for designing a longer MDR-TB regimen remain largely unchanged from those of previous guidance, with the overall goal of having at least four effective second-line medicines and pyrazinamide in the intensive phase [5,23]. The main change has been in the option to include clofazimine and linezolid in the place of $p$-aminosalicylic acid as Group C second-line agents. Ofloxacin is no longer recommended as a fluoroquinolone, while streptomycin may be used if none of the other three injectable agents can be employed. The Group D agents are added to strengthen the regimen or to replace other agents that cannot be used for reasons such as intolerance or drug resistance. The decision to exceed the minimum number of medicines in the regimen (such as the addition of the isoniazid and ethambutol) needs to balance any additional benefit to the risk of incremental harm or interruption as the pill-burden increases. Children with mild disease may be treated without injectable agents. The new data on delamanid led WHO to update its policy on the use of delamanid, which may now also be added to longer MDR-TB regimens for patients aged 6-17 years, under the same conditions as in the interim policy for use of delamanid in adults $[18,24]$. However, new data reviewed on bedaquiline led to no change in policy and this medicine remains indicated only in adults [23]. The medications in Group D3 are only used as a last resort. Clarithromycin and other macrolides are not recommended for MDR-TB treatment anymore.

5) In patients with $M D R / R R-T B$, elective partial lung resection (lobectomy or wedge resection) may be used alongside a recommended MDR-TB regimen.

Remarks: The recommendation is narrow in scope and has no bearing on other possible surgical interventions in TB (e.g. emergency operations or extrathoracic procedures). Patients undergoing more radical pneumonectomy were not observed to have better outcomes than those who did not undergo surgery. The analysis could not provide a refined differentiation of the type of patient who would benefit most from the intervention or the type of intervention that would be most advantageous. The benefits of surgery are likely to depend on the patient subgroups. The effect is expected to be moderate in the average patient considered appropriate for surgery. Despite the unknown magnitude of perioperative complications it was considered that there is an overall net benefit from surgery. Surgery is only to be considered after careful selection of candidates and when there is access to good surgical facilities with experienced surgeons. 


\section{Other comments}

Although the lack of data on the effect of delaying MDR-TB treatment precluded a GRADE recommendation, the GDG agreed that a "good practice statement" was in order and that appropriate therapy needs to be started as early as possible when MDR/RR-TB is diagnosed or strongly suspected.

The 2011 recommendations regarding the testing of TB patients for MDR/RR-TB, the monitoring of treatment response, the duration of longer regimens, the delay in starting antiretroviral therapy in MDR-TB patients with HIV infection and models of care thus continue to apply until future evidence reviews indicate a need for their revision (supplementary table S3) $[5,53]$.

\section{Discussion}

The 2016 revisions to the WHO treatment guidelines for drug-resistant TB signal important changes in policy (table 4). A major departure from the previous "convention" is the possibility to treat many MDR/ RR-TB patients with a 9- to 12-month regimen as an initial option, cutting down the length of treatment by a half or more. In contrast to the individualised longer regimens, the shorter regimen has a more standardised composition which also facilitates logistics and administration. The 2016 revisions have an important impact upon the composition of longer MDR-TB regimens and their application. Importantly for children, there are now options to use delamanid in patients aged 6-17 years and also to avoid injectable agents in mild forms of disease. Finally, an evidence-informed recommendation also recognises the role of partial lung resection surgery in MDR-TB care.

While the shorter MDR-TB regimen has now become the first choice for eligible MDR/RR-TB patients, its application in any given setting will depend primarily upon the prevalence of resistance additional to MDR-TB. Once programmes implement the recommended treatment regimen algorithm (figure 1), the proportion of patients on longer regimens who will have additional resistance will be larger than before;

TABLE 4 Summary of changes made by the 2016 updates to previous World Health Organization (WHO) policy on the treatment of drug-resistant tuberculosis (TB)

The priority questions which were covered by the May 2016 update of the WHO treatment guidelines for drug-resistant TB related to the composition of treatment regimens for multidrug- and rifampicin-resistant TB (MDR/RR-TB); the effectiveness and safety of shorter MDR-TB regimens; the role of elective surgery in MDR-TB management; the treatment of isoniazid-resistant and Mycobacterium bovis tuberculosis; and the impact of delays in starting treatment. From June 2016, the WHO also reviewed additional data on bedaquiline and delamanid.

The main changes in the 2016 recommendations are:

- A second-line treatment is recommended for all patients with rifampicin-resistant tuberculosis, regardless of whether isoniazid resistance is confirmed or not.

- A shorter MDR-TB treatment regimen is conditionally recommended for MDR/RR-TB patients under specific eligibility criteria.

- Recommendations for the treatment of children with MDR/RR-TB are based on a first-ever meta-analysis of individual-level paediatric patient data for treatment outcomes.

- Medicines used in the design of longer MDR-TB treatment regimens are now grouped differently, based upon current evidence on their effectiveness and safety. Clofazimine and linezolid are now considered more important MDR-TB regimen components, while $p$-aminosalicylic acid has been reclassified with agents used only as a last option. Clarithromycin and other macrolides are no longer included as medicines for the treatment of MDR/RR-TB. Delamanid may also be used in patients aged 6-17 years old.

- An evidence-informed recommendation on partial resection lung surgery is now included.

The evidence available on the treatment of isoniazid-resistant TB and on the delay to starting MDR-TB treatment could not address the guideline questions. There were very few published studies on the treatment of $M$. bovis and the regimens differed, precluding any attempt at formulating recommendations of clinical use.

The scope of the 2016 update of MDR-TB treatment policy did not include other aspects of the programmatic management of drug-resistant TB for which no substantive new evidence had emerged since the previous revision. The 2011 recommendations regarding the testing of TB patients for rifampicin resistance, the monitoring of treatment response, the duration of longer regimens, the delay in starting antiretroviral therapy in MDR-TB patients with HIV infection and models of care thus continue to apply until future evidence reviews show a need for revision. No change is made to the recommended use of bedaquiline from those of $2013[20,33]$. 
bedaquiline or delamanid could therefore benefit these patients. With half the MDR-TB patients globally harbouring strains resistant to agents from Groups A or B or both [6], demand for these new medicines is destined to increase markedly from the modest levels being ordered by countries to date.

Given limited experience with the shorter MDR-TB regimen as well as combinations of new and repurposed medicines, WHO has developed a framework to promote the close monitoring and prompt response to adverse drug reactions [35]. Active TB drug safety monitoring and management (aDSM) is intended as an essential component of routine patient monitoring for response to treatment, alongside bacteriological monitoring, which has been integral to TB programmes for many years [54]. Countries have already started to report serious adverse events to a global aDSM database coordinated by WHO/ GTB and the Special Programme for Research and Training in Tropical Diseases hosted by WHO [55]. This database aims to detect signals of unknown or poorly documented harms associated with treatment across pooled data. In addition to aDSM, monitoring for relapse will be important in patients on the shorter MDR-TB regimen until more evidence becomes available on their effectiveness.

The implementation of these guidelines is bound to increase the demand for diagnostics for MDR/RR-TB and XDR-TB. By the end of 2015, more than 500 laboratories in 70 low- and middle-income countries had the capacity to perform LPA technology to diagnose resistance to rifampicin and isoniazid within a couple of days [56]. Such sites are well positioned to extend testing for resistance to fluoroquinolones and second-line injectable agents at a marginal cost. While molecular testing for the rapid detection of $\mathrm{MDR} / \mathrm{RR}-\mathrm{TB}$ and XDR-TB is the preferred diagnostic approach, conventional smear and culture remain important to monitor treatment response [57].

Surgery has been employed in the treatment of TB patients since before the advent of chemotherapy and retains an eminent place in the therapeutic approaches for $\mathrm{TB}$ in some countries. With the unsettling prospect that more patients will present with forms of TB that are practically untreatable with available medicines, the role of surgery deserves to be re-evaluated in different settings. The evidence reviews conducted for the 2016 guidelines update revealed substantial weaknesses in the knowledge base. The data preclude more refined analysis to answer questions of critical relevance to the clinician, such as when best to time surgery, its effects in patients with HIV and how to optimise concomitant medical therapies.

The 2016 update of the WHO drug-resistant TB treatment guidelines rigorously followed the GRADE process for a transparent review of evidence and the formulation of recommendations. It also included one "good practice statement" [58]. Among the overarching gaps identified during this process was the persistent lack of high- or moderate-quality evidence for the interventions of interest. As a result, the certainty in the estimates of effects for all the recommendations was qualified as "very low". Both the strength of a recommendation and the certainty in the evidence upon which the recommendation is based tend to influence the degree to which it is adopted [59]. This underscores the need for more RCTs and for observational series which are better designed and reported, in an effort to limit the confounding and other biases that have characterised many studies of MDR-TB treatment outcomes [41].

The research priorities identified by the GDG largely echoed those shortlisted by other experts of late [60]. Key patient subgroups, such as children, extrapulmonary TB patients and people living with HIV, need to be included in studies or/and study reports showing results stratified by these groups. Adverse events need to be exhaustively collected and uniformly reported to facilitate pooled analysis. Disease markers which are prognostic of treatment response need to be identified urgently and robust assays for their detection developed. RCTs which can guide several aspects of drug-resistant TB treatment are unlikely to materialise in the near future, meaning that results from observational studies will remain important. Therefore, more complete and precise reporting of variables correlated with outcome determinants is needed as these will be important covariates to adjust for when estimating the effect of an intervention. A case in point is surgery, where detailed evidence-informed guidance is very much needed to identify optimal conditions when to operate. Pharmacokinetic studies will be important to optimise dosing and drug safety, especially in childhood $\mathrm{TB}$, pregnancy and in the presence of other comorbidities. The benefits and harms of preventive treatment for contacts of drug-resistant TB need to be investigated urgently, using RCTs. Implementation research is needed to inform end-users on beneficial interventions, on costs and on outcomes such as quality of life.

Stronger policy is likely to convince more implementers to roll out innovative treatment at the scale needed to improve outcomes in patients with drug-resistant TB. The results of the STREAM trial for the shorter regimen, including its bedaquiline arm, and of the phase III trial of delamanid are eagerly awaited to strengthen the existing knowledge base and generate better evidence [61, 62]. Forthcoming reviews of observational series are expected to reopen the discussion on the treatment of isoniazid-resistant TB, as well as on the design of longer MDR-TB regimens and the duration of the intensive and continuation phases. A WHO/GTB expert consultation on the pharmacokinetics and pharmacodynamics of TB medicines in 2017 is expected to provide guidance on dosing, administration and drug-drug interactions. 
The results of drug resistance surveillance for pyrazinamide and fluoroquinolones in TB patients are informing the debate on future diagnostics and treatment [63]. RCTs investigating different medicines for the treatment of contacts of MDR-TB are expected to report findings within a few years [64, 65].

In conclusion, the 2016 updates to the WHO drug-resistant TB treatment guidelines come at a turning point as governments and national authorities align their TB treatment efforts to the End TB Strategy and the Sustainable Development Goals [66,67]. In this process it becomes crucial for policies to be based on the best available science. Adoption of the new recommendations and their implementation at all levels will require the steadfast commitment of state actors, private providers, technical partners, civil society and funding agencies. It will also require social support measures to facilitate patient adherence. The endorsement of leading international professional bodies in pulmonology, such as the European Respiratory Society, the American Thoracic Society, and the International Union Against Tuberculosis and Lung Disease, to these recommendations by will help spread the message faster and wider to $\mathrm{TB}$ practitioners and decision makers in public health worldwide.

\section{Acknowledgements}

This article reproduces primarily the recommendations and contents of the "WHO treatment guidelines for drug-resistant tuberculosis, 2016 update" [25], which were developed according to the requirements of the WHO Guideline Review Committee for evidence gathering, assessment and formulation of recommendations [30]. All authors were involved in the production of these guidelines and participated at different stages to the writing of this manuscript. The authors are indebted to the many workers who contributed as part of the Guideline Development Group, the External Review Group, the evidence reviewers, the WHO Guideline Steering Group, the WHO Guideline Review Committee, the WHO TB Regional Advisers as well as the staff of national programmes and technical agencies who made data available for the evidence reviews (full list of individuals at [25]). The revision of the guidelines was made possible by funding from the US Agency for International Development (USAID) and USAID was also a major provider of salary support to the WHO authors involved in this article. No specific funding was provided for the adaptation, writing and submission of the article, which was done in the usual course of work of the authors involved. E. Harausz declares that the views expressed in this article are those of the author and should not be construed to represent the positions of the US Army or the US Dept of Defense.

\section{References}

1 General Assembly of the United Nations. High-level meeting on antimicrobial resistance. 2016. www.un.org/pga/ 71/2016/09/21/press-release-hl-meeting-on-antimicrobial-resistance Date last accessed: November 7, 2016.

2 Zignol M, Dean AS, Falzon D, et al. Twenty years of global surveillance of antituberculosis-drug resistance. $N$ Engl J Med 2016; 375: 1081-1089.

3 Wright A, Zignol M, Van Deun A, et al. Epidemiology of antituberculosis drug resistance 2002-07: an updated analysis of the Global Project on Anti-Tuberculosis Drug Resistance Surveillance. Lancet 2009; 373: 1861-1873

4 WHO. Guidelines for treatment of tuberculosis. 4th edn (WHO/HTM/TB/2009.420). 2009. http://whqlibdoc.who. int/publications/2010/9789241547833_eng.pdf Date last accessed: November 7, 2016.

5 Falzon D, Jaramillo E, Schünemann HJ, et al. WHO guidelines for the programmatic management of drug-resistant tuberculosis: 2011 update. Eur Respir J 2011; 38: 516-528.

6 WHO. Global tuberculosis report 2016 (WHO/HTM/TB/2016.13). 2016. www.who.int/tb/publications/global report/en/ Date last accessed: November 7, 2016.

7 Falzon D, Mirzayev F, Wares F, et al. Multidrug-resistant tuberculosis around the world: what progress has been made? Eur Respir J 2015; 45: 150-160.

8 Médecins Sans Frontières, Stop TB Partnership. Out of Step 2015. TB Policies in 24 Countries. A survey of diagnostic and treatment practices. 2015. www.msfaccess.org/sites/default/files/MSF_assets/TB/Docs/TB_report_ Out_of_Step_ENG_2015.pdf Date last accessed: November 7, 2016.

9 WHO. Towards universal access to diagnosis and treatment of multidrug-resistant and extensively drug-resistant tuberculosis by 2015. WHO progress report 2011 (WHO/HTM/TB/2011.3). 2011. http://whqlibdoc.who.int/ publications/2011/9789241501330_eng.pdf Date last accessed: November 7, 2016.

10 Dye C, Williams BG, Espinal MA, et al. Erasing the world's slow stain: strategies to beat multidrug-resistant tuberculosis. Science 2002; 295: 2042-2046.

11 WHO. Resolution WHA62.15. Prevention and control of multidrug-resistant tuberculosis and extensively drug-resistant tuberculosis. (WHA62/2009/REC/1). 2009. http://apps.who.int/gb/ebwha/pdf_files/WHA62-REC1/ WHA62_REC1-en.pdf Date last accessed: November 7, 2016.

12 Uplekar M, Weil D, Lönnroth K, et al. WHO's new end TB strategy. Lancet 2015; 385: 1799-1801.

13 WHO. Guidelines for the management of drug-resistant tuberculosis (WHO/TB/96.210 (Rev.1)). 1997. http://apps. who.int/iris/bitstream/10665/63465/1/WHO_TB_96.210_\%28Rev.1\%29.pdf Date last accessed: November 7, 2016.

14 WHO. Guidelines for establishing DOTS-Plus pilot projects for the management of multidrug-resistant tuberculosis (MDR-TB) (WHO/CDS/TB/2000.279). 2000. http://apps.who.int/iris/bitstream/10665/66368/1/WHO_ CDS_TB_2000.279_eng.pdf Date last accessed: November 7, 2016.

15 WHO. Guidelines for the programmatic management of drug-resistant tuberculosis. 1st edn (WHO/HTM/TB/ 2006.361). 2006. http://apps.who.int/iris/bitstream/10665/246249/2/9789241546959-eng.pdf Date last accessed: November 7, 2016.

16 WHO. Policy guidance on drug susceptibility testing (DST) of second-line anti-tuberculosis drugs (WHO/HTM/ TB/2008.392). 2008. http://whqlibdoc.who.int/hq/2008/WHO_HTM_TB_2008.392_eng.pdf Date last accessed: November 7, 2016.

17 WHO. Guidelines for the programmatic management of drug-resistant tuberculosis, Emergency update 2008 (WHO/HTM/TB/2008.402). 2008. http://whqlibdoc.who.int/publications/2008/9789241547581_eng.pdf Date last accessed: November 7, 2016. 
18 WHO. Guidelines for the programmatic management of drug-resistant tuberculosis, 2011 Update (WHO/HTM/ TB/2011.6). 2011. http://whqlibdoc.who.int/publications/2011/9789241501583_eng.pdf Date last accessed: November 7, 2016

19 WHO. Rapid implementation of the Xpert MTB/RIF diagnostic test: technical and operational 'How-to'; practical considerations. 2011. whqlibdoc.who.int/publications/2011/9789241501569_eng.pdf Date last accessed: November 7, 2016.

20 WHO. The use of bedaquiline in the treatment of multidrug-resistant tuberculosis. Interim policy guidance (WHO/HTM/TB/2013.6). 2013. http://apps.who.int/iris/bitstream/10665/84879/1/9789241505482_eng.pdf Date last accessed: November 7, 2016.

21 WHO. The use of delamanid in the treatment of multidrug-resistant tuberculosis. Interim policy guidance (WHO/ HTM/TB/2014.23). 2014. http://apps.who.int/iris/bitstream/10665/137334/1/WHO_HTM_TB_2014.23_eng.pdf Date last accessed: November 7, 2016.

22 WHO. Automated real-time nucleic acid amplification technology for rapid and simultaneous detection of tuberculosis and rifampicin resistance: Xpert MTB/RIF assay for the diagnosis of pulmonary and extrapulmonary TB in adults and children. Policy update (WHO/HTM/TB/2013.16). 2013. http://apps.who.int/iris/bitstream/ 10665/112472/1/9789241506335_eng.pdf Date last accessed: November 7, 2016.

23 WHO. Companion handbook to the WHO guidelines for the programmatic management of drug-resistant tuberculosis (WHO/HTM/TB/2014.11). 2015. http://apps.who.int/iris/bitstream/10665/130918/1/9789241548809_ eng.pdf Date last accessed: November 7, 2016.

24 WHO. Xpert MTB/RIF implementation manual: technical and operational 'How-to'; practical considerations (WHO/HTM/TB/2014.1). 2014. http://apps.who.int/iris/bitstream/10665/112469/1/9789241506700_eng.pdf Date last accessed: November 7, 2016.

25 WHO. WHO treatment guidelines for drug-resistant tuberculosis, 2016 update (WHO/HTM/TB/2016.04). 2016. www.who.int/tb/areas-of-work/drug-resistant-tb/treatment/resources/en/ Date last accessed: November 7, 2016.

26 WHO. Implementing tuberculosis diagnostics: a policy framework (WHO/HTM/TB/2015.11). 2015. http://apps. who.int/iris/bitstream/10665/162712/1/9789241508612_eng.pdf Date last accessed: November 7, 2016.

27 WHO. The use of delamanid in the treatment of multidrug-resistant tuberculosis in children and adolescents Interim policy guidance (WHO/HTM/TB/2016.14). 2016. http://apps.who.int/iris/bitstream/10665/250614/1/ 9789241549899-eng.pdf Date last accessed: November 7, 2016.

28 WHO. The use of molecular line probe assays for the detection of resistance to second-line anti-tuberculosis drugs. Policy guidance (WHO/HTM/TB/2016.07). 2016. www.who.int/tb/publications/lpa-mdr-diagnostics/en/ Date last accessed: November 7, 2016.

29 Guyatt GH, Oxman AD, Vist GE, et al. GRADE: an emerging consensus on rating quality of evidence and strength of recommendations. BMJ 2008; 336: 924-926.

30 WHO. WHO handbook for guideline development. 2nd edn. 2014. http://apps.who.int/medicinedocs/documents/ s22083en/s22083en.pdf Date last accessed: November 7, 2016.

31 Guyatt G, Oxman AD, Akl EA, et al. GRADE guidelines: 1. Introduction - GRADE evidence profiles and summary of findings tables. J Clin Epidemiol 2011; 64: 383-394.

32 Schünemann HJ, Wiercioch W, Etxeandia I, et al. Guidelines 2.0: systematic development of a comprehensive checklist for a successful guideline enterprise. CMAJ 2014; 186: E123-E142.

33 WHO. Guideline Development Group (GDG) Meetings: revision of the interim policy on bedaquiline for MDR-TB treatment and special session on delamanid use in children. 2016. www.who.int/tb/areas-of-work/ treatment/gdg_bedaquiline_delamanid/en/ Date last accessed: November 7, 2016.

34 The Cochrane Collaboration. Cochrane Handbook for Systematic Reviews of Interventions. 2011. http://handbook. cochrane.org/ Date last accessed: November 7, 2016.

35 WHO. Active tuberculosis drug-safety monitoring and management (aDSM). Framework for implementation (WHO/HTM/TB/2015.28). 2015. http://apps.who.int/iris/bitstream/10665/204465/1/WHO_HTM_TB_2015.28_ eng.pdf Date last accessed: November 7, 2016.

36 WHO. WHO treatment guidelines for drug-resistant tuberculosis, 2016 update (WHO/HTM/TB/2016.04) Online Annexes 4, 5, 6. 2016. http://apps.who.int/iris/bitstream/10665/250125/5/9789241549639-webannexes-eng.pdf Date last accessed: November 7, 2016.

37 Van Deun A, Maug AKJ, Salim MAH, et al. Short, highly effective, and inexpensive standardized treatment of multidrug-resistant tuberculosis. Am J Respir Crit Care Med 2010; 182: 684-692.

38 International Union Against Tuberculosis and Lung Disease. MDR-TB study in Francophone Africa. 2013. http:// liverpool.worldlunghealth.org/updates/francophone-africa-announces-breakthrough-results-at-union-conference Date last accessed: February 13, 2017.

39 Kuaban C, Noeske J, Rieder HL, et al. High effectiveness of a 12-month regimen for MDR-TB patients in Cameroon. Int J Tuberc Lung Dis 2015; 19: 517-524.

40 Piubello A, Harouna SH, Souleymane MB, et al. High cure rate with standardised short-course multidrug-resistant tuberculosis treatment in Niger: no relapses. Int J Tuberc Lung Dis 2014; 18: 1188-1194.

41 Ahuja SD, Ashkin D, Avendano M, et al. Multidrug resistant pulmonary tuberculosis treatment regimens and patient outcomes: an individual patient data meta-analysis of 9,153 patients. PLoS Med 2012; 9: e1001300.

42 Aung K, van Deun A, Declercq E, et al. Successful '9-month Bangladesh regimen' for multidrug resistant tuberculosis among over 500 consecutive patients. Int J Tuberc Lung Dis 2014; 18: 1180-1187.

43 Lee M, Lee J, Carroll MW, et al. Linezolid for treatment of chronic extensively drug-resistant tuberculosis. $N$ Engl J Med 2012; 367: 1508-1518.

44 Tang S, Yao L, Hao X, et al. Efficacy, safety and tolerability of linezolid for the treatment of XDR-TB: a study in China. Eur Respir J 2015; 45: 161-170.

45 Tang S, Yao L, Hao X, et al. Clofazimine for the treatment of multidrug-resistant tuberculosis: prospective, multicenter, randomized controlled study in China. Clin Infect Dis 2015; 60: 1361-1367.

46 Winters N, Butler-Laporte G, Menzies D. Efficacy and safety of World Health Organization group 5 drugs for multidrug-resistant tuberculosis treatment. Eur Respir J 2015; 46: 1461-1470.

47 Fox GJ, Mitnick CD, Benedetti A, et al. Surgery as an adjunctive treatment for multidrug-resistant tuberculosis: an individual patient data metaanalysis. Clin Infect Dis 2016; 62: 887-895. 

tuberculosis: a systematic review and meta-analysis. BMC Infect Dis 2016; 16: 262. Harris RC, Grandjean L, Martin LJ, et al. The effect of early versus late treatment initiation after diagnosis on the outcomes of patients treated for multidrug-resistant tuberculosis: a systematic review. BMC Infect Dis 2016 ; 16 : 193.

50 Case definition for extensively drug-resistant tuberculosis. Wkly Epidemiol Rec 2006; 81: 408.

51 WHO. Frequently asked questions about the implementation of the new WHO recommendation on the use of the shorter MDR-TB regimen under programmatic conditions. 2016. www.who.int/entity/tb/areas-of-work/drug-resistanttb/treatment/FAQshorter_MDR_regimen.pdf Date last accessed: February 13, 2017.

52 MSF Field Research. Research Protocol - Effectiveness of a simplified short regimen for Multidrug Resistant Tuberculosis treatment in Karakalpakstan, Uzbekistan. 2014. http://fieldresearch.msf.org/msf/handle/10144/322296 Date last accessed: November 7, 2016.

53 WHO. Consolidated guidelines on the use of antiretroviral drugs for treating and preventing HIV infection. Recommendations for a public health approach. 2nd edn. 2016. http://apps.who.int/iris/bitstream/10665/208825/1/ 9789241549684_eng.pdf Date last accessed: November 7, 2016.

54 WHO. Definitions and reporting framework for tuberculosis - 2013 revision (WHO/HTM/TB/2013.2). 2013. www.who.int/iris/bitstream/10665/79199/1/9789241505345_eng.pdf Date last accessed: November 7, 2016.

55 WHO. WHO global database for TB active drug safety monitoring home page. www.who.int/tdr/research/tb_hiv/ adsm/en/ Date last accessed: November 7, 2016.

56 WHO. TB data home page. www.who.int/tb/country/en/ Date last accessed: November 7, 2016.

57 Mitnick CD, White RA, Lu C, et al. Multidrug-resistant tuberculosis treatment failure detection depends on monitoring interval and microbiological method. Eur Respir J 2016; 48: 1160-1170.

58 Guyatt GH, Schünemann HJ, Djulbegovic B, et al. Guideline panels should not GRADE good practice statements. J Clin Epidemiol 2015; 68: 597-600.

59 Nasser SMU, Cooke G, Kranzer K, et al. Strength of recommendations in WHO guidelines using GRADE was associated with uptake in national policy. J Clin Epidemiol 2015; 68: 703-707.

60 Mitnick CD, Rodriguez CA, Hatton ML, et al. Programmatic management of drug-resistant tuberculosis: an updated research agenda. PLoS One 2016; 11: e0155968.

61 Nunn AJ, Rusen ID, Van Deun A, et al. Evaluation of a standardized treatment regimen of anti-tuberculosis drugs for patients with multi-drug-resistant tuberculosis (STREAM): study protocol for a randomized controlled trial. Trials 2014; 15: 353.

62 ClinicalTrials.gov. Safety and efficacy trial of delamanid for 6 months in patients with multidrug resistant tuberculosis. https://clinicaltrials.gov/ct2/show/NCT01424670 Date last accessed: November 7, 2016.

63 Zignol M, Dean AS, Alikhanova N, et al. Population-based resistance of Mycobacterium tuberculosis isolates to pyrazinamide and fluoroquinolones: results from a multicountry surveillance project. Lancet Infect Dis 2016; 16: 1185-1192.

64 Stellenbosch University Dept of Paediatrics and Child Health. A phase III cluster randomised placebo-controlled trial to assess the efficacy of preventive therapy in child and adolescent contacts of MDR-TB (TB-CHAMP) http://gtr.rcuk.ac.uk/projects?ref=MR/M007340/1 Date last accessed: November 7, 2016.

65 ANZCTR. The V-QUIN MDR TRIAL: a randomized controlled trial of six months of daily levofloxacin for the prevention of tuberculosis among household contacts of patients with multi-drug resistant tuberculosis https:// anzctr.org.au/Trial/Registration/TrialReview.aspx?id=369817 Date last accessed: November 7, 2016.

66 WHO. Implementing the End TB Strategy: the essentials (WHO/HTM/TB/2015.31). 2015. www.who.int/tb/ publications/2015/end_tb_essential.pdf Date last accessed: November 7, 2016.

67 UN Department of Economic and Social Affairs. Sustainable Development Goals. 2015. https://sustainabledevelopment. un.org/sdgs Date last accessed: November 7, 2016. 